\title{
Current density, chemical shifts and aromaticity ${ }^{\dagger}$
}

\author{
P. W. Fowler, ${ }^{1 *}$ E. Steiner, ${ }^{1}$ R. W. A. Havenith ${ }^{2}$ and L. W. Jenneskens ${ }^{3}$ \\ ${ }^{1}$ Department of Chemistry, University of Exeter, Stocker Road, Exeter EX4 4QD, UK \\ 2 Debye Institute, Theoretical Chemistry Group, Utrecht University, Padualaan 14, 3584 CH Utrecht, The Netherlands \\ ${ }^{3}$ Debye Institute, Department of Physical Organic Chemistry, Utrecht University, Padualaan 8, 3584 CH Utrecht, The Netherlands \\ Received 6 April 2004; Revised 14 April 2004; Accepted 14 April 2004
}

\begin{abstract}
The intimate link between chemical shifts and magnetic criteria for aromaticity prompts a search for detailed understanding of patterns of current density induced in $\pi$ systems by external magnetic fields. Conceptual and practical advantages of calculation of current densities with a specific method of distribution of origin of vector potential, the ipsocentric choice, where the induced current density at each point is calculated with that point as origin, are outlined. This choice leads uniquely to canonical molecular orbital contributions that are free of unphysical occupied-occupied mixing. Characteristic magnetic response of delocalized $\pi$ systems is then effectively restricted to the activity of a small number of frontier electrons, governed by simple symmetry and node-counting rules, and readily visualized in current density maps. Localized orbitals for $\sigma$ systems can also be used, again eliminating occupied-occupied mixing. For integrated properties (magnetizability and nuclear shieldings), the ipsocentric method gives, in a well-defined sense, the orbital contributions that are best for purposes of interpretation. The general theory is illustrated by maps for a set of annelated pentalenes; the known benzopentalene (1) and 1,2:4,5-dibenzopentalene (2), the still unknown isomer of 2, 1,2:5,6-dibenzopentalene (3), and cyclopent $[b, c]$ acenaphthylene (4), an unknown isomer of pyracylene, all of which consist of fusions of formally aromatic and anti-aromatic $\pi$-conjugated systems. Copyright $\odot 2004$ John Wiley \& Sons, Ltd.
\end{abstract}

KEYWORDS: NMR; current density; chemical shifts; anisotropy

\section{INTRODUCTION}

As with charge density in the study of electric properties, current density is central to the understanding of molecular magnetic properties and underpins the theory and practice of NMR spectroscopy. Although the mapping of charge density in molecules has a long history, it is only recently that the construction of maps of induced current density has become a standard theoretical tool. This seems all the more surprising when it is recalled that the existence of a specific feature in the induced current density, the ring current, is taken as the basis for the (magnetic) criterion of aromaticity.

Since the pioneering work of Pauling ${ }^{1}$ and London ${ }^{2}$ in the 1930s, ring current has been invoked in the explanation of the characteristic magnetic susceptibilities of aromatics ${ }^{3}$ and, since the classic paper of Pople, ${ }^{4}$ ring currents have been inferred from the anomalous ${ }^{1} \mathrm{H}$ shifts in aromatic molecules. The ability to sustain a diatropic ring current is taken as the signature of aromaticity in a cyclic molecule; ${ }^{5}$ in the same way, paratropic ring current betokens anti-aromaticity. ${ }^{6}$ This magnetic criterion is enshrined in the NICS method, ${ }^{7}$ where a fictitious 'nuclear shielding' is calculated for a point at or

${ }^{\dagger}$ Dedicated to Professor M. Barfield on the occasion of his 70th birthday.

*Correspondence to: P. W. Fowler, Department of Chemistry, University of Exeter, Stocker Road, Exeter EX4 4QD, UK.

E-mail: pwfowler@ex.ac.uk near a ring centre as a numerical indicator of the existence, strength and sense of the current and hence a measure of aromaticity.

A more direct approach to ring-current aromaticity, the inspection of the full spatial variation of current density induced in an unsaturated planar system by a perpendicular magnetic field, was for a long time blocked by the problem of gauge dependence in computed magnetic properties. Although the decomposition of properties such as magnetizability and nuclear magnetic shielding into diamagnetic (classical free-electron circulation) and paramagnetic (quantum-mechanical response to molecular nonuniformity) contributions is in a sense arbitrary, in that the balance of these contributions depends on the choice of origin, total response properties should be invariant to this choice. In practice, in calculations with a finite basis and with a single origin of vector potential, computed properties are found to be far from invariant, and the underlying current density is poorly represented. Substantial progress in quantitative calculation of global properties was made as it became clear that distribution of origin of vector potential was advantageous, ${ }^{8-10}$ and the breakthrough in the treatment of the basic underlying property, the current density, was achieved when Keith and Bader ${ }^{11}$ proposed the ultimate 'democratic' distribution of origin - a treatment in which an individual origin was used for calculation of current density at every point in space. This ansatz, which in its simplest version is often called ipsocentric ${ }^{12}$ (in that current density 
at any one point is calculated with that point as origin), has important practical and conceptual advantages. ${ }^{12-16}$

The present paper summarizes this approach and shows how ipsocentric calculations lead to a tractable qualitative picture of magnetic aromaticity in which ring currents can be attributed to features of frontier orbital structure and hence can be understood qualitatively and also calculated quantitatively. This leads to an interpretation of chemical shifts at nuclei and at the notional NICS centres and an interpretation of the survival and extinction of currents in clamped systems, polycycles and heterocycles. Some new calculations on benzo-fused pentalenes are used to illustrate the power of the method and cast light on these problematic systems.

The structure of the paper is as follows. First, the ipsocentric calculation of current density is briefly outlined and it is shown how this leads to a particular orbital model of induced current. Connections with molecular symmetry are then established, leading to rules of thumb for aromaticity and anti-aromaticity and giving the basis of a physical interpretation of orbital contributions. Finally, the machinery of the ipsocentric method is applied to a set of molecules of long-standing interest in the study of aromaticity and its limits, the benzopentalenes. These species incorporate nominally anti-aromatic pentalene and aromatic benzene or naphthalene, giving scope for interplay of local and global aromaticity/anti-aromaticity. Limited experimental data are available for comparison. Benzopentalene (1) (cyclopent $[a]$ indene) dimerizes above $-70^{\circ} \mathrm{C}$ in $\mathrm{CD}_{2} \mathrm{Cl}_{2}$ solution, but a monomer ${ }^{1} \mathrm{H}$ NMR spectrum is available, recorded at $-70{ }^{\circ} \mathrm{C}$. ${ }^{17}$ There is a low-resolution ${ }^{1} \mathrm{H}$ NMR spectrum for the more symmetrical dibenzopentalene [1,2:4,5-dibenzopentalene (2)]. ${ }^{18}$ The isomeric dibenzopentalene 1,2:5,6-dibenzopentalene (3) is so far unknown, as is the species cyclopent $[b, c]$ acenaphthylene (4), formally a fusion of pentalene and naphthalene moieties, which has been invoked as a pivotal transient intermediate in high temperature, gas-phase reactions including the unimolecular rearrangement ${ }^{19}$ of pyracylene and the interconversions of ethynyl-substituted acenaphthylenes and bisethynylnaphthalenes. ${ }^{20}$ Computed maps ${ }^{21}$ of current density for 4 show clear separation of aromatic and anti-aromatic regions. Comparison with $\mathbf{1}$ to $\mathbf{3}$ will show whether the magnetic identity of the pentalene subunit survives annelation in more general situations.

\section{INDUCED CURRENT DENSITY}

The theory of the induced current density has been described before, and detailed discussions of the ipsocentric approach to gauge distribution have been published. ${ }^{12,22,23}$ Here we summarize the main points of the derivation of the magnetic response, in the particular form that leads to a natural definition of molecular orbital contributions. ${ }^{12,23}$ We consider the closed-shell ground state of an $N$-electron system, with doubly-occupied orbitals $\psi_{n}(n=1,2, \ldots, N / 2)$ and unoccupied orbitals $\psi_{p}(p>N / 2)$, all of which are eigenfunctions of a one-electron Hamiltonian $\hat{H}_{0}$ with corresponding eigenvalues (orbital energies) $\varepsilon_{n}, \varepsilon_{p}$. In our calculations, the $\psi_{n}$ are Hartree-Fock orbitals and firstorder corrections are calculated with coupled-Hartree-Fock theory, but could equally be obtained from empirical $\mathrm{MO}$ theories or DFT. For a constant uniform magnetic field $\mathbf{B}$, the Hamiltonian is, to first order in $\mathbf{B}$ :

$$
\hat{H}=\hat{H}_{0}+\frac{e^{2}}{2 m_{\mathrm{e}}} \hat{\mathbf{I}}(\mathbf{d}) \cdot \mathbf{B}
$$

where $\hat{\mathbf{l}}(\mathbf{d})=(\mathbf{r}-\mathbf{d}) \times \mathbf{p}$ is the angular momentum operator for rotation about the 'gauge origin' $\mathbf{d}$ of the magnetic vector potential $\mathbf{A}=1 / 2 \mathbf{B} \times(\mathbf{r}-\mathbf{d})$ and $\hat{\mathbf{p}}=-i \hbar \nabla$ is the linear momentum operator. Then the first-order induced current density, $\mathbf{j}^{(1)}(\mathbf{r})$, is a sum of terms, one for each doubly occupied orbital $\psi_{n}$ :

$$
\begin{aligned}
\mathbf{j}_{n}(\mathbf{r})= & -\frac{e^{2}}{m_{\mathrm{e}}} \mathbf{B} \times(\mathbf{r}-\mathbf{d}) \psi_{n}(\mathbf{r})^{2} \\
& +\frac{2 i e \hbar}{m_{\mathrm{e}}}\left[\psi_{n}(\mathbf{r}) \nabla \psi_{n}^{(1)}(\mathbf{r})-\psi_{n}^{(1)}(\mathbf{r}) \nabla \psi_{n}(\mathbf{r})\right]
\end{aligned}
$$

The first term on the right-hand side, the 'diamagnetic current density', $\mathbf{j}_{n}^{(\text {dia) }}(\mathbf{r})$, depends on the unperturbed orbital density; the second, the 'paramagnetic current density', $\mathbf{j}_{n}^{\text {(para) }}(\mathbf{r})$, depends also on the first-order correction, $\psi_{n}^{(1)}$. This separation into diamagnetic and paramagnetic parts has no special physical significance as terms can be rebalanced within a constant total, by choice of gauge origin, which in principle changes all orbital current densities $\mathbf{j}_{n}(\mathbf{r})$, as well as their diamagnetic/paramagnetic breakdown, even in a complete basis set.

To simplify the discussion, we consider the one-electron Hamiltonian to be constructed from the usual kinetic and local potential energy operators. Orbital degeneracy, selfconsistency, exchange and other non-local corrections ${ }^{24}$ complicate the argument, but do not change its essentials. ${ }^{23}$ The first-order correction to each occupied orbital $\psi_{n}$ is then a sum over excitations from that orbital to unoccupied orbitals $\psi_{p}(p>N / 2):^{12}$

$$
\psi_{n}^{(1)}(\mathbf{r})=-\frac{e}{2 m_{\mathrm{e}}} \sum_{p>N / 2} \psi_{p}(\mathbf{r}) \frac{\left\langle\psi_{p}|\hat{\mathbf{I}}(\mathbf{d}) \cdot \mathbf{B}| \psi_{n}\right\rangle}{\varepsilon_{p}-\varepsilon_{n}}
$$

The key feature of this sum is that it excludes any possibility of occupied-occupied mixings. By rewriting the angular momentum operator for rotation about $\mathbf{d}$ as $\hat{\mathbf{l}}(\mathbf{d})=\hat{\mathbf{l}}(\mathbf{0})-\mathbf{d} \times \hat{\mathbf{p}}$, where $\hat{\mathbf{l}}(\mathbf{0})$ refers to rotation about the origin of coordinates, the first-order correction can be split into two sums, corresponding to paramagnetic and diatropic terms, each still excluding any reference to occupied-occupied mixings. We have

$$
\begin{aligned}
\psi_{n}^{(1)}(\mathbf{r})= & -\frac{e}{2 m_{\mathrm{e}}}\left[\sum_{p>N / 2} \psi_{p}(\mathbf{r}) \frac{\left\langle\psi_{p}|\hat{\mathbf{I}}(\mathbf{0})| \psi_{n}\right\rangle}{\varepsilon_{p}-\varepsilon_{n}}\right] \cdot \mathbf{B} \\
& +\frac{e}{2 m_{\mathrm{e}}}\left[\mathbf{d} \times \sum_{p>N / 2} \psi_{p}(\mathbf{r}) \frac{\left\langle\psi_{p}|\hat{\mathbf{p}}| \psi_{n}\right\rangle}{\varepsilon_{p}-\varepsilon_{n}}\right] \cdot \mathbf{B}
\end{aligned}
$$

In the first sum, $\psi_{n}^{\text {(para) }}(\mathbf{r})$, the quantity $\left\langle\psi_{p}|\hat{1}(\mathbf{0})| \psi_{\mathrm{n}}\right\rangle$ is an angular momentum transition moment, modulated by 
the orbital energy difference $\varepsilon_{p}-\varepsilon_{n}$, which determines accessibility of unoccupied orbital $\psi_{p}$ from occupied $\psi_{n}$ via a rotational transition. Similarly in the second sum, $\psi_{n}^{\text {(dia) }}(\mathbf{r})$, the numerator $\left\langle\psi_{p}|\hat{\mathbf{p}}| \psi_{\mathrm{n}}\right\rangle$ is a linear-momentum transition moment, modulated by $\varepsilon_{p}-\varepsilon_{n}$, which determines the accessibility of unoccupied $\psi_{p}$ from occupied $\psi_{n}$ via a translational transition. Exclusion of occupied-to-occupied orbital transitions from the sums follows from antisymmetry properties of the $N$-electron wavefunction.

The essential insight of Keith and Bader ${ }^{11}$ was that the gauge origin need not be the same for all positions $\mathbf{r}$, but may be any finite single-valued parametric function of position, $\mathbf{d}(\mathbf{r})$, with the induced current density $\mathbf{j}^{(1)}(\mathbf{r})$ at each point $\mathbf{r}$ computed with respect to $\mathbf{d}(\mathbf{r})$. The ipsocentric choice, $\mathbf{d}-\mathbf{r}=\mathbf{0}$, leads to formal annihilation of the diamagnetic term $\mathbf{j}_{n}^{\text {(dia) }}(\mathbf{r})$ for every orbital, hence one of its acronyms, CTOCD-DZ ${ }^{14,15}$ (continuous transformation of origin for current density, diamagnetic-zero).

Gauge-origin dependence for choices of arbitrary gauge origin $\mathbf{d}$ can be expressed with respect to the ipsocentric current density as reference. The full derivation of the result is given with all mathematical detail elsewhere, ${ }^{23}$ and is summarized briefly here. First, an auxiliary function is defined, through which non-physical translational occupied-occupied mixings are explicitly reinstated in $\mathbf{j}_{n}^{(\mathrm{dia})}(\mathbf{r})$; this is shown to give rise to gauge-origin dependence of orbital contributions, with strength proportional to the displacement of $\mathbf{r}$ from $\mathbf{d}$. The $\mathbf{d}$-dependent terms cancel when orbital contributions with the same $\mathbf{d}$ function are summed, so that, unlike the orbital contributions, the total current density in a complete basis is always independent of the choice of gauge origin. These redundant non-physical terms are eliminated from individual orbital current densities if and only if the diamagnetic term is itself eliminated, that is, if and only if the formulation is ipsocentric. In any other formulation, the contribution of an occupied-to-occupied orbital transition $\psi_{n} \rightarrow \psi_{m}$ in $\mathbf{j}_{n}$ is cancelled by the contribution of the reversed transition $\psi_{m} \rightarrow \psi_{n}$ in $\mathbf{j}_{m}$. Thus, for example, if the first is a diamagnetic contribution, the second is an opposing paramagnetic contribution of equal magnitude.

In general, the cancelling contributions can become arbitrarily large, and this removes all hope of a convincing interpretation of a total property in terms of the orbitals. Such contributions must be rigorously excluded if orbital contributions are to make physical sense. The ipsocentric orbital contributions formulation have specific physical significance in that, orbital by orbital, the current density at each point $\mathbf{r}$ is the local response of the electron distribution to the twisting force of the magnetic field. Ipsocentric orbital contributions are sums of local magnetic dipole transition moments that determine the accessibility of unoccupied orbital $\psi_{p}$ from orbital $\psi_{n}$, weighted by the orbital energy difference.

For $\pi$ systems, maps of current density are usually plotted as projections of the current density induced by a perpendicular external magnetic field; decomposition into contributions from $\sigma$ and $\pi$ subsystems and into more detailed orbitalby-orbital maps can then be made as required. The standard theoretical level for calculation of the maps is with optimized geometries at the restricted Hartree-Fock (RHF) level, in the $6-31 G^{* *}$ basis set, with calculation of the induced current density by solution of the coupled Hartree-Fock equations in the ipsocentric description. Integration over all space of the current density in combination with appropriate operators leads to magnetizability (magnetic susceptibility) and magnetic shieldings. These quantities all depend linearly on the induced current density. As this current density is a sum of orbital contributions, so are the integrated magnetic response properties, and the ipsocentric approach again gives an economical interpretation of response properties, even if in practice other variants of the method (such as CTOCD-PZ2 ${ }^{15}$ ) and/or larger basis sets are used to perform the integrated calculations.

\section{IMPLICATIONS FOR INTERPRETATION SYMMETRY RULES AND MONOCYCLES}

The form of the ipsocentric first-order wavefunction, with the restriction of the sum to occupied-virtual transitions, and the occurrence of orbital energy differences in the denominators, suggests that, other things being equal, current density will arise from the activity of relatively few electrons near the HOMO energy, where the gap between occupied and empty orbitals is smallest. Truly delocalized systems have this feature, with contributions concentrated in the frontier canonical orbitals; more localized systems show a spread of contributions from a wider range of orbitals. The number of contributing orbitals and the 'open' or 'closed' nature of the orbital circulations can be used to give an intrinsic magnetic criterion of localizability of orbitals. ${ }^{22}$

A very general consequence of the form of the firstorder wavefunction is that since the response of the electrons to the external field is determined by transition moments of operators with well-defined symmetries, there should be clear selection rules for the contributions, ${ }^{12}$ and a strong connection between aspects of the symmetry of a molecule and its aromaticity/anti-aromaticity on the magnetic criterion. This connection is most easily seen for planar carbocycles, ${ }^{13}$ the subjects of the classical Hückel $4 n+2 / 4 n$ rules, but it has implications for all systems with exact or approximate symmetry.

Consider a planar conjugated system in which the ring currents are induced by a magnetic field at right angles to the molecular $(x, y)$ plane. Symmetry determines whether a transition from occupied orbital $n$ to unoccupied orbital $m$ can contribute to the current density, spatial distribution of the orbitals determines whether an allowed transition moment will be large or small and the difference in orbital energies affects the relative weight of a given transition moment in the total current. If $G$ is the point group of the field-free molecule, then a contribution can be classified as conventionally diamagnetic or paramagnetic (with respect to an origin that is unshifted under all operations of $G$ ) using the symmetries in $G$. If $\Gamma\left(\psi_{n}\right), \Gamma\left(R_{z}\right)$ and $\Gamma\left(T_{x}, T_{y}\right)$ denote representations of an orbital (or degenerate set of orbitals), rotation around the field direction and the two translations at right angles to the field, then, since angular and linear momentum operators transform as rotations and translations, respectively, the transition $\psi_{n} \rightarrow \psi_{m}$ makes 
a conventionally diamagnetic (diatropic) contribution to current density if the product $\Gamma\left(\psi_{n}\right) \times \Gamma\left(T_{x}, T_{y}\right) \times \Gamma\left(\psi_{m}\right)$ contains a totally symmetric component, a conventionally paramagnetic (paratropic) contribution to current density if the product $\Gamma\left(\psi_{n}\right) \times \Gamma\left(R_{z}\right) \times \Gamma\left(\psi_{m}\right)$ contains a totally symmetric component, a mixed contribution if both products have such a component and no contribution if neither product has one.

Under Hückel approximations for a ring of $N$ atoms with equal Coulomb parameters $\alpha$ connected by bonds of equal resonance parameter $\beta$, the $\pi$ molecular orbital energies are given by the well-known Frost-circle construction, ${ }^{25}$ where energies correspond to the heights of vertices of an inscribed $N$-gon inscribed standing on one corner in a circle of radius $2 \beta$ drawn about the origin, and orbitals are sine/cosine combinations associated with an angular momentum quantum number $\lambda=0,1, \ldots,(N / 2)$ ( $N$ even), $\ldots,(N-1) / 2$ ( $N$ odd). Closed-shell configurations occur at 'aromatic' counts of $4 n+2=2,6,10, \ldots$ and, in the same approximation, open shells with two electrons in two degenerate orbitals occur at 'anti-aromatic' counts $4 n=4,8, \ldots \pi$-electrons.

For monocycles, the outline of the ipsocentric description of currents is then as follows. In the closed-shell $4 n+2$ configurations, the occupied orbitals form complete shells. Since in-plane rotation mixes only orbitals of equal $\lambda$, it cannot produce occupied-to-virtual transitions. On the other hand, in-plane translational operators introduce (or delete) nodes and therefore mix orbitals on adjacent rungs of the energy ladder that differ by \pm 1 in $\lambda$. The sole occupied-tovirtual translational transitions in the $(4 n+2)$ system are therefore HOMO to LUMO, and so, in a $(4 n+2)$-electron monocycle, the ring current arises solely from mobility of the HOMO electrons and is wholly diamagnetic. Except when $n=0$, the HOMO is doubly degenerate and the $(4 n+2)$ system has four-electron diamagnetism. ${ }^{13}$ This conclusion survives at the $a b$ initio level [see Fig. 1(a)].

In $4 n$ systems, the $\pi$ system has half-filling of the angular momentum shell of highest $\lambda$. In full $D_{N h}$ symmetry, this would lead to an open-shell $e^{2}$ configuration, but distortion lifts this degeneracy, for example, in a bondalternated $D_{(N / 2) h}$ structure. In the reduced symmetry, the HOMO-LUMO pair splits into non-degenerate components related by a rotational transition with a small energy denominator. This HOMO-LUMO term dominates the sum over states, producing a strong two-electron paramagnetic $\pi$ ring current. ${ }^{13}$ Other rotational and translational transitions introduced by the lowering in symmetry are less important in small ring systems. Again, this conclusion survives at the ab initio level [see Fig. 1(b)].

This account of the origin of diatropic and paratropic ring currents in the frontier electronic structure of monocycles is essentially graph theoretical in nature-the selection rules hinge on node-counting and angular-momentum projection rather than exact point-group symmetry, and they can therefore be expected to survive in less symmetrical situations as more-or-less strict propensity rules. Currents are predicted to persist under a lowering of symmetry that introduces splittings without altering nodal character as (a)
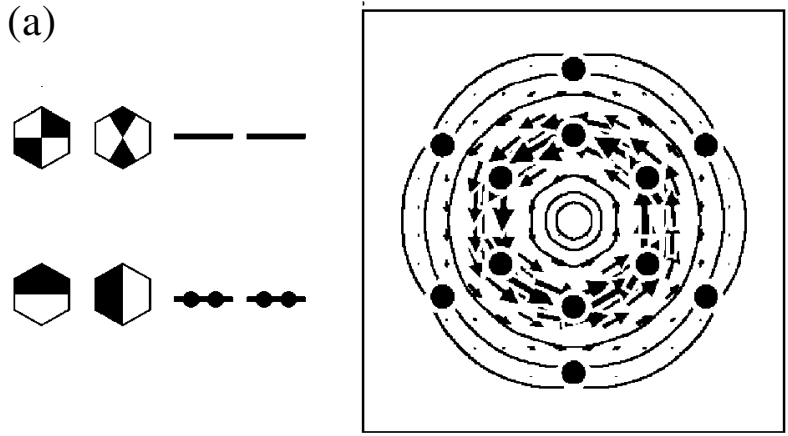

(b)
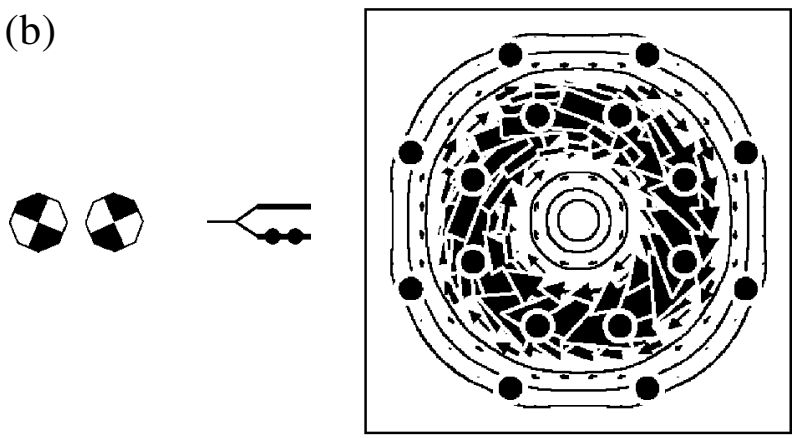

Figure 1. Maps of $\pi$ ring current in monocycles and the $\pi-\pi^{*}$ transitions that produce them. For (a) benzene and (b) cyclooctatetraene (COT) in a constrained planar geometry, the figure shows the map of $\pi$ current density induced by a perpendicular magnetic field, plotted in a plane $1 a_{0}$ above that of the nuclei, calculated with the ipsocentric method in the $6-31 G^{* *}$ basis at the best RHF planar geometry for that basis, ${ }^{12,13}$ with a schematic representation of the main orbital contribution. In benzene, the transition is node increasing and the current diatropic; in COT, the transition is node preserving and the current paratropic.

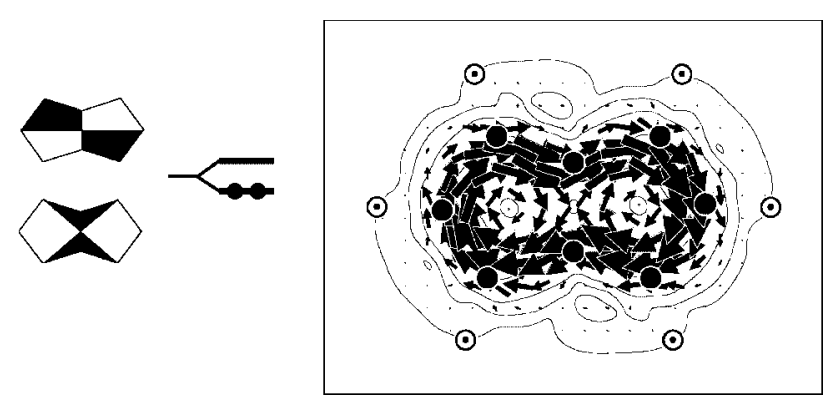

Figure 2. Map of $\pi$ ring current in pentalene, showing the induced $\pi$ current density in the conventions of Fig. 1, at the $\left(C_{2 h}\right)$ RHF optimized geometry of this bicyclic molecule, together with a schematic representation of the main orbital contribution. The HOMO-LUMO transition, descended from the same $\pi-\pi^{*}$ transition in planar COT, preserves node count and the $\pi$ current is therefore paratropic.

in, e.g., carbon-rich heterocycles such as $C_{2 v}$ pyridine, or under purely geometric distortion of a central monocycle, as in 'clamped' benzene and cyclooctatetraene (COT) systems where the annelated clamping groups are saturated and introduce only $\sigma / \sigma^{*}$ orbitals around the $\pi / \pi^{*}$ frontier. ${ }^{26}$ 




$1\left(\mathrm{C}_{s}\right)$

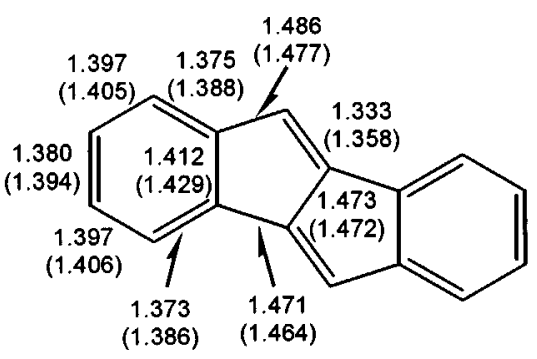

$2\left(\mathrm{C}_{2 h}\right)$

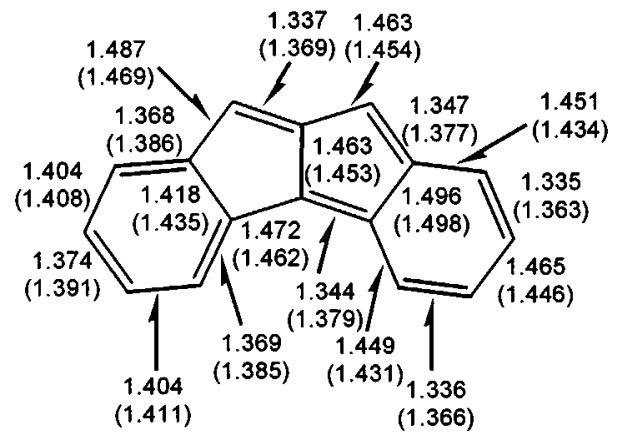

$3\left(\mathrm{C}_{s}\right)$

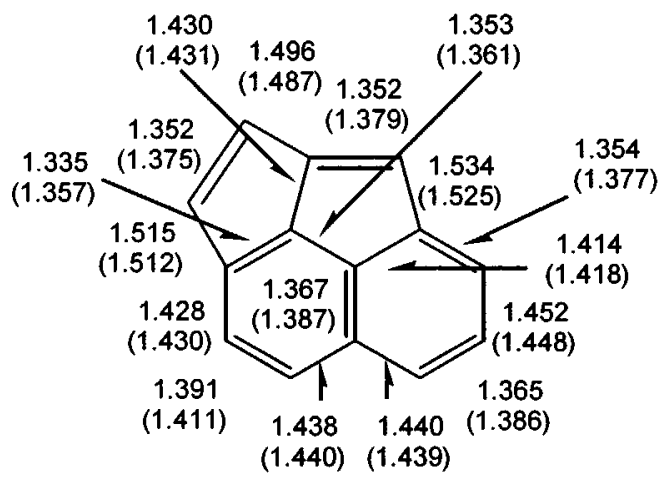

$4\left(\mathrm{C}_{1} \mathrm{RHF}, \mathrm{C}_{s}\right.$ DFT $)$
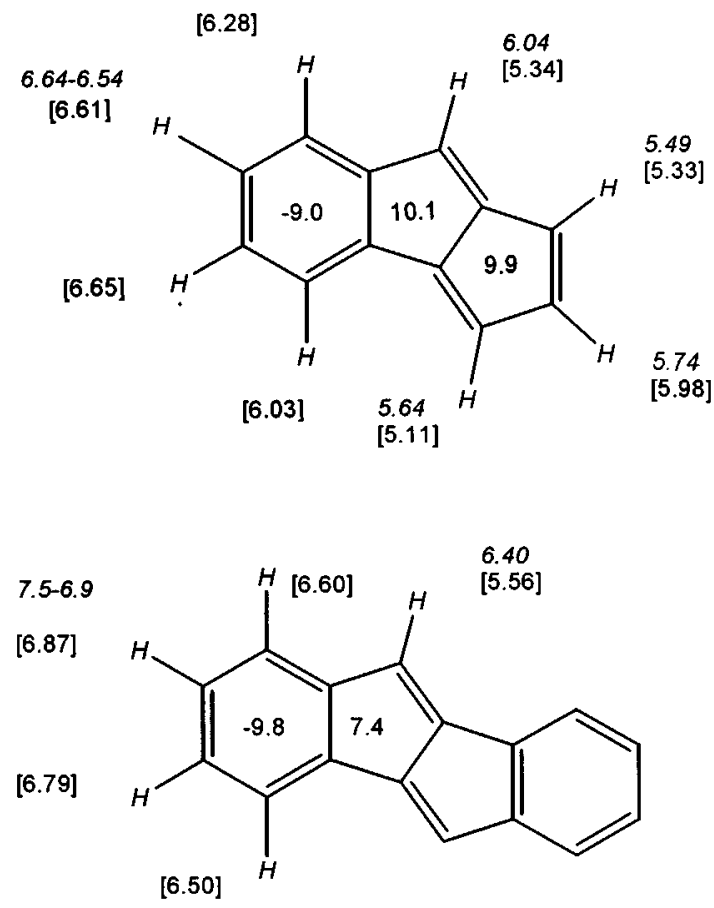

[6.15]
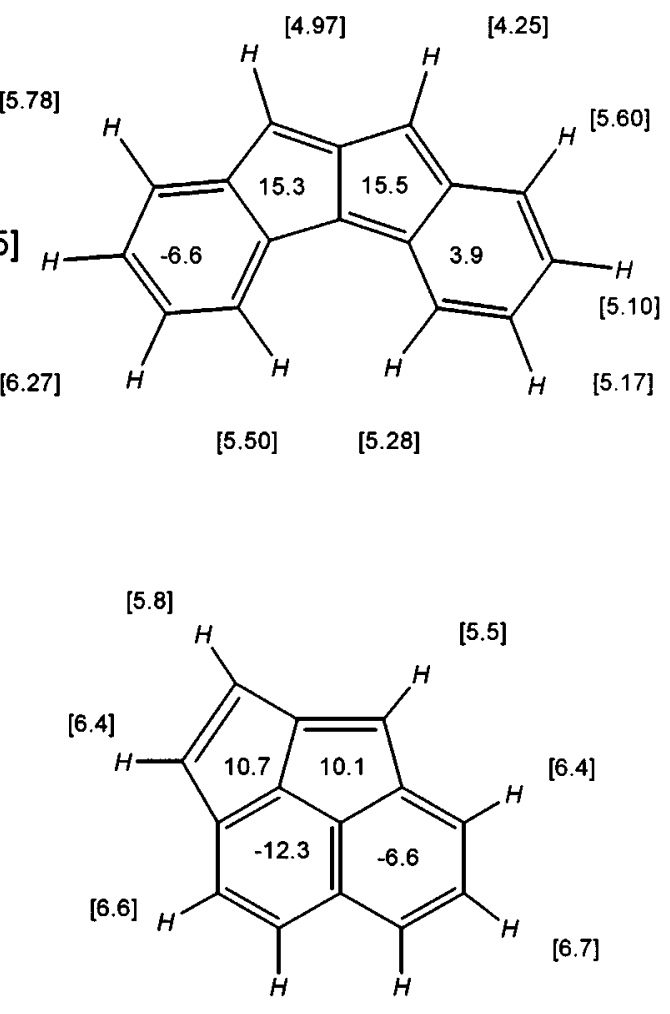

[6.9]

[6.7]

Figure 3. Geometries and integrated magnetic properties of annelated pentalenes. On the left are the RHF/6-31G** (unbracketed) and B3LYP/6-31G** (bracketed) optimized bond lengths of benzopentalene [1 $\left.\left(C_{s}\right)\right],{ }^{17} 1,2: 4,5$-dibenzopentalene $\left[2\left(C_{2 h}\right)\right],{ }^{18}$ 1,2:5,6-dibenzopentalene [ $\left.3\left(C_{s}\right)\right]$, and cyclopenta[b,c]acenaphthylene [ $4\left(C_{1}\right.$ at the RHF level, $C_{s}$ at the DFT level)]. On the right are the chemical shifts for the same molecules: computed CTOCD-PZ2/6-31G**//RHF/6-31G** ${ }^{1} \mathrm{H}$ NMR and NICS shifts are shown in square brackets. Available experimental ${ }^{1} \mathrm{H}$ NMR chemical shifts are shown in italics. 




(c)

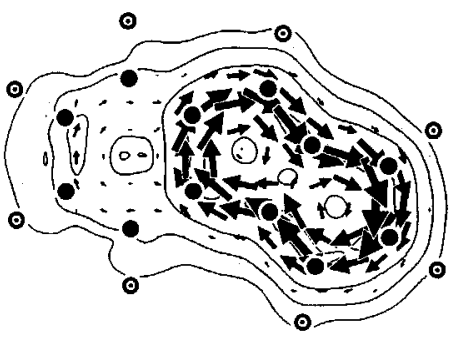

$6 a^{\prime \prime}$

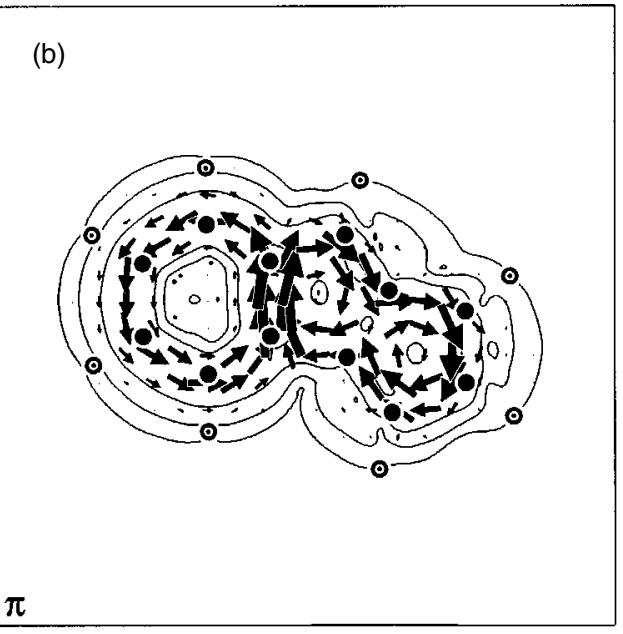

(d)

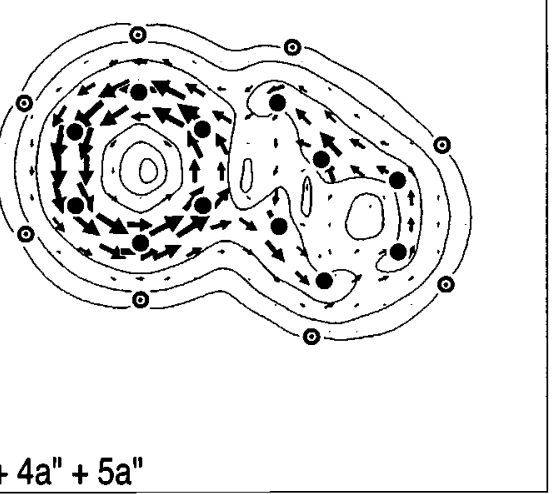

$3 a^{\prime \prime}+4 a^{\prime \prime}+5 a^{\prime \prime}$

Figure 4. Maps of induced ring current in benzopentalene $\left[1\left(C_{s}\right)\right.$ ]. Plotting conventions as in Fig. 1. The maps show (a) the contribution of all $(\sigma+\pi)$ orbitals; (b) the contribution of all $\pi$ orbitals; (c) the contribution of the HOMO; and (d) the contribution of the three $\pi$ orbitals lying immediately below the HOMO.

Systems such as the trannulenes, where strict $\sigma / \pi$ separation has been lost, but where the nodal structure of the $\pi$ system persists in the manifold of radial orbitals also obey these rules. $^{27}$ On the other hand, perturbations which alter the HOMO and LUMO structure, as when an unsaturated clamping group introduces new orbitals at the frontier, ${ }^{26}$ or when the $\pi$ orbitals of a heterocycle localize under large electronegativity gradients, as in $\mathrm{B}_{3} \mathrm{~N}_{3} \mathrm{H}_{6},{ }^{22,28}$ will typically lead to loss of global ring current.

Considerations of symmetry and nodal character can be used to understand ring and perimeter currents in polycyclic systems, as will be demonstrated in detail for the benzofused pentalenes below. In pentalene itself, with $4 n=8 \pi$ electrons on eight centres, HOMO and LUMO correspond to originally degenerate $\lambda=2$ non-bonding levels of the octagonal cycle. Stabilization of one level by adding a crosslink to the cycle leaves the other as a formally non-bonding LUMO, separated from the HOMO by a small gap, and so pentalene has a strong paratropic perimeter circulation (see Fig. 2) for the same reasons that COT in constrained planar geometries supports an (anti-aromatic) paratropic ring current. ${ }^{29}$ Similarly, the diatropic perimeter current of naphthalene is a descendant of the ring current of the planar form of a [10] annulene..$^{29,30}$ These results are compatible with simple electron counting, but follow specifically from the preservation of frontier-orbital topology on symmetrical cross-linking. Electron counting alone would not be sufficient if this match of orbitals were absent. Systems such as corannulene and coronene, circulene topologies supporting counter-rotating currents, ${ }^{31,32}$ illustrate the need to go beyond simple electron bookkeeping.

A detailed analysis of the nuclear (and nucleusindependent) shieldings in benzene has been published elsewhere, ${ }^{23}$ and has shown that the ipsocentric description cast in terms of appropriate molecular orbitals (delocalized canonical orbitals for $\pi$, localized orbitals for $\sigma$ ) leads to the most economical description of the orbital origins of the characteristic components of shielding and magnetizability tensors. One striking conclusion of the study is that, even for this simple molecular system, whereas the current density map allows interpretation of shieldings (and indeed can predict them quantitatively on integration), it can be risky to try to infer the current pattern from a shielding value, particularly if that value is itself an isotropic average, a single measured shift or a NICS. Reproducibility of experimental chemical shifts is an important test of theoretical current 

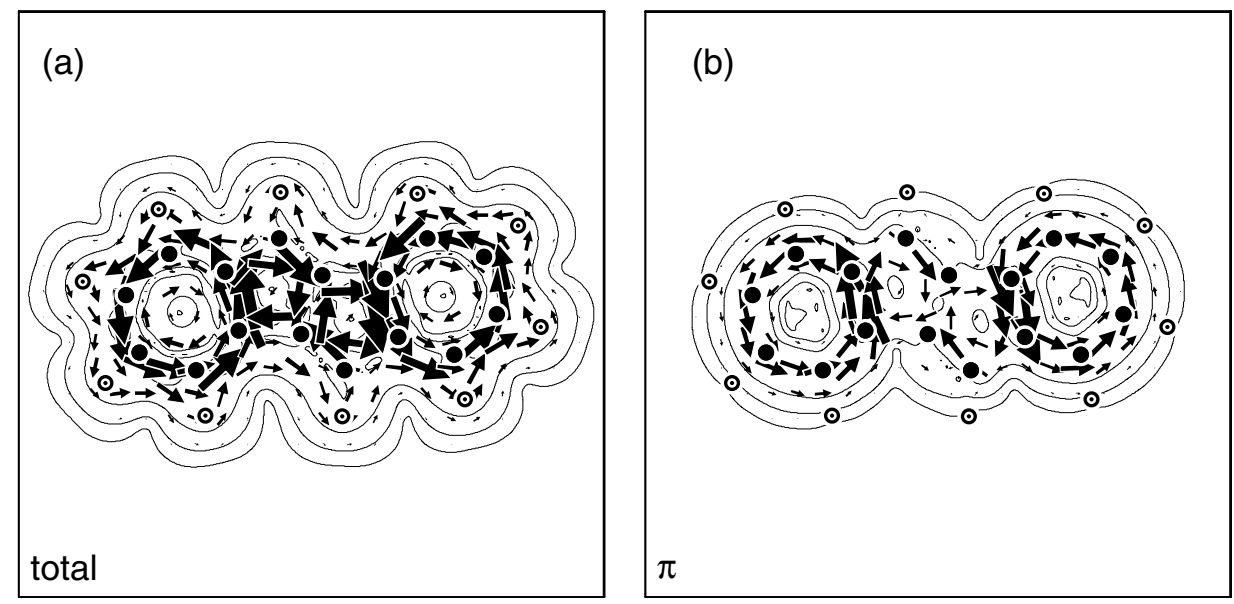

(c)



Figure 5. Maps of ring current induced in 1,2:4,5-dibenzopentalene [2 $\left.\left(C_{2 h}\right)\right]$. Plotting conventions as in Fig. 1. The maps show (a) the contribution of all $(\sigma+\pi)$ orbitals; (b) the contribution of all $\pi$ orbitals; (c) the contribution of the HOMO; and (d) the contribution of the three $\pi$ orbitals lying immediately below the HOMO.

densities, which they have been shown to pass, ${ }^{21,33,34}$ but inspection of maps computed at even a modest level may reveal a rich variety of behaviour that is obscured by the integration and averaging processes.

\section{CURRENT DENSITY MAPS FOR THE BENZOPENTALENES}

The geometries of the benzopentalenes 1-3 were optimized at the RHF /6-31G** and B3LYP/6-31G** levels of theory using GAMESS-UK, ${ }^{35}$ and the RHF optimized structures were used in the computation of current-density maps with the SYSMO program. ${ }^{36}$ Molecules 1-3 each show no essential difference in geometry or symmetry between RHF and DFT structures. For benzopentalene (1), 1,2:4,5dibenzopentalene (2) and 1,2:5,6-dibenzopentalene (3), the optimized structures belong to $C_{s}, C_{2 h}$ and $C_{s}$ point groups, respectively. Hessian calculations were used to check that all structures were all genuine minima. Although the point groups found for $\mathbf{1}$ and $\mathbf{2}$ are maximal, that of 3 is reduced from $C_{2 v}$, as could be expected from simple considerations since a Hückel calculation shows the bond-bond polarizability matrix for this molecule to have one large eigenvalue $\left(\left|\beta \lambda^{\pi}{ }_{\max }\right|=1.834\right)$, just exceeding the threshold value $^{37}$ for realization of $\pi$ distortivity. In the distortion implied by the maximal bond-polarizability eigenvector, 1,2:5,6-dibenzopentalene (3) possesses two nonidentical annelated hexagons, one with three mobile double bonds and one with two fixed double bonds. The loss of symmetry in 3 persists at the DFT level; the optimization in the $6-31 \mathrm{G}^{* *}$ basis with the B3LYP functional also yields a $C_{s}$ geometry. This picture is supported by a survey of the computed bond lengths in the series $1-3$. Whereas in the cases of $\mathbf{1}$ and $\mathbf{2}$ the hexagons are still recognizably benzenoid, in the case of $\mathbf{3}$ two distinct hexagons are discernible, i.e. one with typical benzenoid carbon-carbon bond lengths and one with typical bond alternation as found in polyenes (see Fig. 3). Figure 3 also includes the $C_{1}$ RHF/6-31G** geometry of 4, which loses its small distortions and returns to $C_{s}$ symmetry at the B3LYP/6-31G** level; ${ }^{21}$ both sets of bond lengths are shown in Fig.3.

Current-density maps for systems $\mathbf{1}-\mathbf{3}$ were computed in the ipsocentric CTOCD-DZ approach described above, and are shown in Figs 4-6. Similar maps are available for $4 .{ }^{21}$ Nuclear magnetic shielding tensors were computed using the same basis in the CTOCD-PZ2 approach ${ }^{15}$ for the ${ }^{1} \mathrm{H}$ sites, and also for the geometric centres of all rings. Mean ${ }^{1} \mathrm{H}$ shieldings $\sigma\left({ }^{1} \mathrm{H}\right)$ were converted to shifts $\delta\left({ }^{1} \mathrm{H}\right)$ through the approximate relation $\delta=30.8-10^{6} \times \sigma^{15,33,34}$ and mean shieldings at ring centres converted to $\mathrm{NICS}^{7}$ values by 


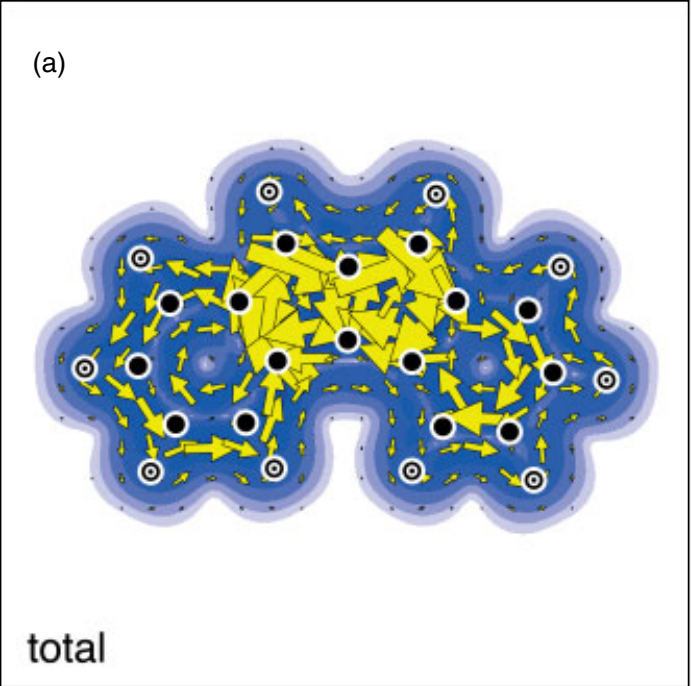

(c)

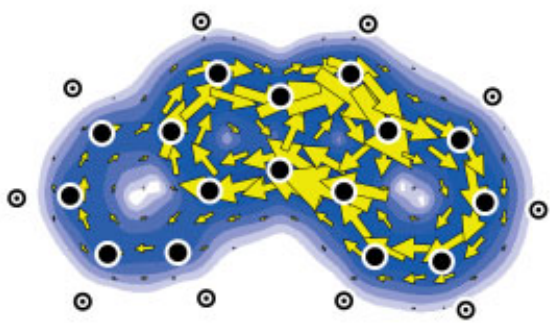

8a" (b)

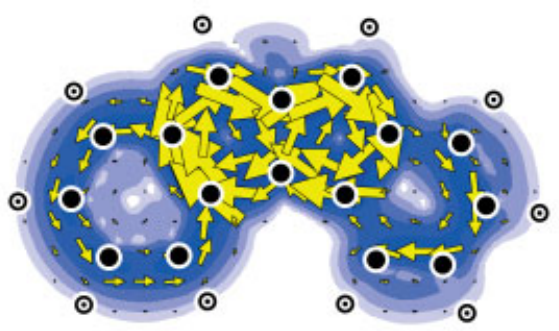

$\pi$

(d)



$5 a^{\prime \prime}+6 a^{\prime \prime}$

Figure 6. Maps of ring current induced in 1,2:5,6-dibenzopentalene [3 $\left(C_{s}\right)$ ]. Plotting conventions as in Fig. 1. The maps show (a) the contribution of all $(\sigma+\pi)$ orbitals; (b) the contribution of all $\pi$ orbitals; (c) the contribution of the HOMO; and (d) the contribution of the two most significant $\pi$ orbitals below the HOMO.

change of sign. The various calculated shifts are shown with the molecular structures in Fig. 3, with the published data ${ }^{20}$ for 4 for comparison. The use of the PZ2 technique for the calculation of shieldings rather than the $D Z$ (ipsocentric) method used for the diaplayed maps perhaps requires some comment. It has been shown ${ }^{15}$ that of the two methods, both of which distribute fully the origin of vector potential, it is PZ2 that gives the integrated properties that converge more rapidly to the basis-set limit. However, maps from $P Z(2)$ and DZ approaches are visually similar, apart from the regions close to nuclei, ${ }^{16}$ and they therefore give the same messages about the presence or absence of global ring currents, delocalization and aromaticity. Integration to give nuclear shielding is sensitive to regions near the nucleus, hence the technical superiority of $P Z 2$ for this task. For the maps, DZ is used for its economy, but most importantly for the unique advantages of its orbital-by-orbital partition. ${ }^{23}$

In each set of current-density maps, the total $(\sigma+\pi)$ current is shown, and also the pure $\pi$ contribution to it, projected in the plotting plane 1 Bohr $\left(1 a_{0}\right)$ above that of the nuclei. The maps give a direct and clear visualization of the differences amongst the various annelated pentalenes 1-3.

Thus, in benzopentalene (1, Fig. 4), annelation of a single benzene ring to the pentalene core has produced a molecule that retains distinct diatropic and paratropic regions, with pentalene keeping its identity as a locally anti-aromatic $8 \pi$-electron circuit that supports a paratropic ring current, and benzene preserving its locally aromatic character. Current strength can be estimated by inspection of the largest arrows in the regions over the various CC bonds: over the perimeter of the benzene ring in 1, these vary from 0.050 to 0.075 (in atomic units of current density per unit inducing field), or from 63 to $95 \%$ of the value (0.080 a.u.) calculated in the same way for benzene itself; the pentalene unit has paratropic currents of similar strength (0.062 to 0.090 a.u.) around the perimeter, but the strongest current is over the benzene-pentalene fusion bond, where the two circulations reinforce to give 0.012 a.u, or $140 \%$ of the benzene standard value. Further 

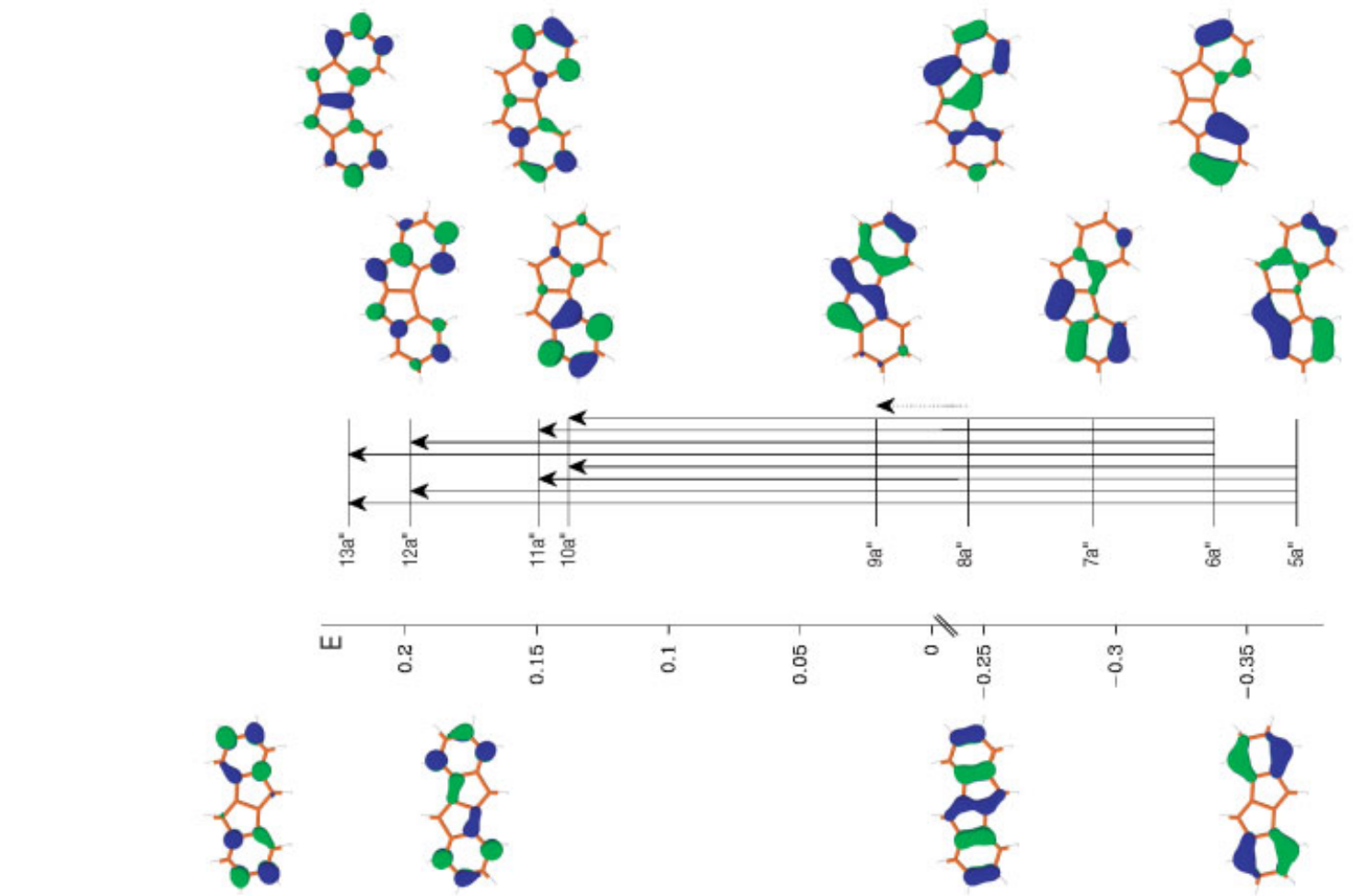

30
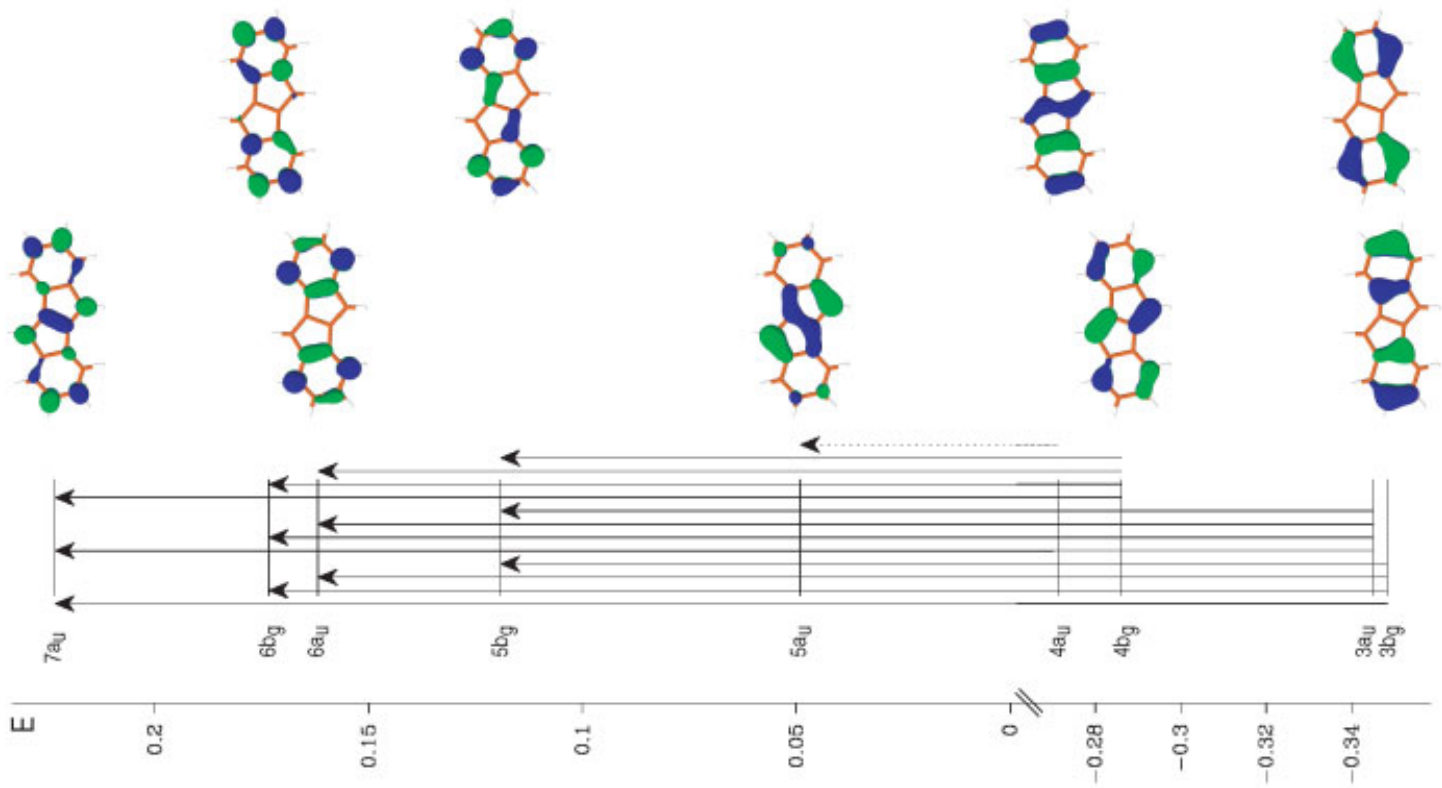

20
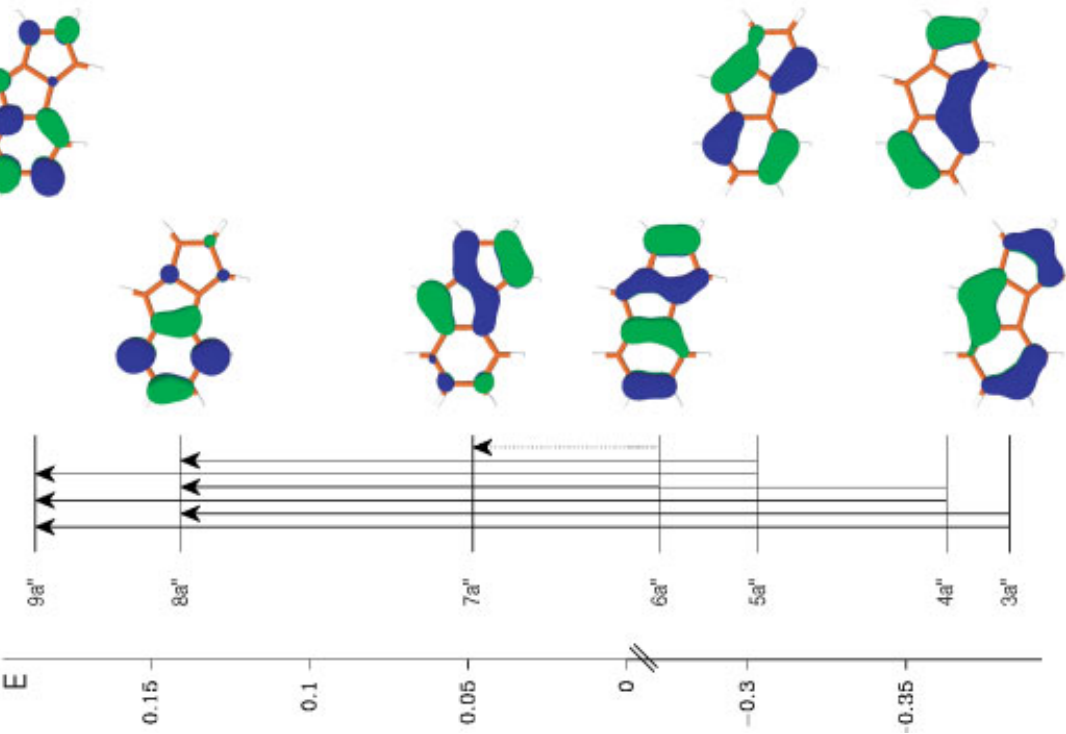
analysis of the orbital contributions shows that the pentalene circulation is a feature arising essentially from the response of the two HOMO electrons $\left(6 a^{\prime \prime}\right)$, whereas the benzene circulation is part of a general diatropic perimeter current, with significant contributions from at least three orbitals $\left[\right.$ HOMO-1 $\left(5 a^{\prime \prime}\right)+$ HOMO-2 $\left(4 a^{\prime \prime}\right)+$ HOMO-3 $\left.\left(3 a^{\prime \prime}\right)\right]$ below the HOMO. The partition of current into two regions of countercirculation is consistent with and explains the trend of the NICS values for the three rings. In the low $C_{s}$ point group of this system, the symmetry forms of the diatropic/paratropic selection rules are not helpful, since the relevant operators are trivially symmetric: $\Gamma\left(T_{x}, T_{y}\right)=\Gamma\left(R_{z}\right)=a^{\prime}$, but the current is still understandable in orbital node-counting terms. The HOMO-LUMO transition involves local rotation of the nodal structure over the pentalene moiety, whereas transitions from HOMO-1 $\left(5 a^{\prime \prime}\right)$, HOMO-2 $\left(4 a^{\prime \prime}\right)$, HOMO-3 $\left(3 a^{\prime \prime}\right)$ to $\mathrm{LUMO}+1\left(8 a^{\prime \prime}\right), \mathrm{LUMO}+2\left(9 a^{\prime \prime}\right), \mathrm{LUMO}+3\left(10 a^{\prime \prime}\right)$ all involve an increase of one node in the benzene ring [Fig. 7(a)].

The observation of distinct diatropic and paratropic ring current in $\mathbf{1}$ is also reflected in the computed ${ }^{1} \mathrm{H}$ NMR chemical shifts computed by appropriate integration of its induced current density. For this purpose, bearing in mind the modest size of the $6-31 \mathrm{G}^{* *}$ basis, the paramagnetic zero (PZ2) variant of CTOCD ${ }^{15}$ as implemented in SYSMO ${ }^{36}$ was used. Previously, we have shown that the CTOCD-PZ2 approach gives ${ }^{1} \mathrm{H}$ NMR chemical shifts that are of sufficient quality for structural assignment. ${ }^{21,33,34}$ In agreement with the limited experimental ${ }^{1} \mathrm{H}$ NMR shift data for $\mathbf{1},{ }^{17}$ the pentalene-type hydrogens are all shifted upfield and positioned in the olefinic region. In contrast, the benzenoid hydrogens of $\mathbf{1}$ remain positioned in the aromatic region (Fig. 3). This difference between rings is further substantiated by the CTOCD-PZ2 nucleus-independent chemical shifts [NICS $(0.0 \AA)$ ] calculated in the ring centres of the distinct five- and six-membered rings of $\mathbf{1}$ (Fig. 3).

The maps for 1,2:4,5-dibenzopentalene (2) show a further development on the same theme (Fig. 5). Now the current pattern has three distinct regions, with a central paratropic pentalene bordered by two diatropic benzene rings. Currents in the perimeter are of similar strength (70-100\%) to those in benzene; in the benzene-pentalene fusion bonds where the sub-unit currents reinforce, they reach $125 \%$ of the benzene value. The pentalene current is again a two- $\pi$-electron contribution (from the $4 a_{u} \mathrm{HOMO}$ ), characteristic of the HOMO-LUMO transition of an anti-aromatic $4 n$ cycle; local benzene currents again arise as part of a general diatropic perimeter circulation that has significant contributions from several of the orbitals that lie just below the HOMO. The local rotational character of the HOMO-LUMO transition can again be seen from the nodal structure of the frontier orbitals of 2 [Fig. 7(b)].

The presence of the distinct diatropic and paratropic ring currents in the hexagons and pentagons, respectively, of $\mathbf{2}$ is again reflected in the computed ${ }^{1} \mathrm{H}$ NMR chemical shifts computed by integration of the induced current density. NICS values for individual rings are again readily explained by the regional character of the current patterns.
This straightforward interpretation of currents as superpositions of pentalene-like and benzene-like contributions in the case of benzopentalene (1) and 1,2:4,5-dibenzopentalene (2) breaks down for 1,2:5,6-dibenzopentalene (3), where one of the benzene rings has been subsumed into a strong paratropic current that extends beyond the pentalene rings. The extended paratropic unit is again a $4 n$ cycle, now comprising 12 carbon centres, and its strong current arises from the $8 a^{\prime \prime}$ HOMO of 3; and the weaker diatropic current on the 'aromatic' benzene ring has contributions from HOMO-2 $\left(6 a^{\prime \prime}\right)$ and HOMO-3 $\left(5 a^{\prime \prime}\right)$, and from other frontier orbitals. Reduced (positive and negative) NICS values are consistent with this overall pattern. In fact, both the current-density maps and NICS values show that one of the hexagons lacks benzenoid character. Further analysis in terms of localized $\pi$ orbitals could prove illuminating.

The computed CTOCD-PZ2 ${ }^{1} \mathrm{H}$ NMR chemical shifts for 3 obtained by integration of induced current density (Fig. 3) show that the hydrogens of the pentalene and one of the hexagon are markedly shifted upfield. In contrast, the hydrogens of the other hexagon are positioned downfield. The computed NICS values are consistent with the latter hexagon sustaining a diatropic ring current and the former supporting an only weakly paratropic current.

Finally, we note that cyclopent $[b, c]$ acenaphthylene (4), which consists of a pentalene annelated to a naphthalene, is similar in behaviour to $\mathbf{1}$ and $\mathbf{2}$, having a well-defined pentalene circulation and a diatropic counter-circulation in the 'aromatic' remainder of the molecule (see Fig. 8). The orbital origin of these currents has been described elsewhere, ${ }^{21}$ and is again consistent with the nodal characteristics of the frontier orbitals. In fact, a detailed analysis of computed current-density maps shows that 4 constitutes an annelated pentalene according to the magnetic criterion of aromaticity, and provides an explanation for the markedly different NICS values in the two 'naphthalene-like' hexagons of 4 (Fig. 3).

\section{CONCLUSION}

This discussion of a single family of polycyclic molecules shows the qualitative insight into the patterns of delocalization and current flow that can be gained from ipsocentric mapping of induced current density. Given the modest computational cost, and clear underlying chemical rationale in



Figure 8. Map of $\pi$ ring current induced in cyclopenta[b,c]acenaphthylene $\left[\mathbf{4}\left(C_{s}\right)\right]$. Plotting conventions as in Fig. 1. 
terms of orbitals, energies, nodes and symmetries, it can be expected that plotting of current-density maps will become a standard tool for the resolution of debates about aromaticity and for the interpretation of the otherwise opaque numerical values provided by chemical shift measurement and calculation.

\section{Acknowledgements}

L.W.J. acknowledges financial support from the Royal Society of Chemistry's Journals Grants for International Authors Programme (0301433). R.W.A.H. acknowledges financial support from The Netherlands Organization for Scientific Research (NWO), grant 700.53.401.

\section{REFERENCES}

1. Pauling L. J. Chem. Phys. 1936; 4: 673.

2. London F. J. Phys. Radium 1937; 8: 397.

3. Dauben HJ, Wilson JD, Laity JL. In Nonbenzenoid Aromatic II, Snyder JP (ed). Academic Press: New York, 1971; 167.

4. Pople JA. J. Chem. Phys. 1956; 24: 1111.

5. Elvidge JA, Jackman LM. J. Chem. Soc. 1961; 859.

6. Longuet-Higgins HC. In Aromaticity. Special Publication No. 21, Chemical Society: London, 1967; 109-110.

7. Schleyer PvR, Maerker C, Dransfeld A, Jiao H, van Eikema Hommes NJR. J. Am. Chem. Soc. 1996; 118: 6317.

8. Ditchfield R. Mol. Phys. 1974; $27: 789$.

9. Kutzelnigg W. Isr. J. Chem. 1980; 19: 193.

10. Schindler M, Kutzelnigg W. J. Chem. Phys. 1982; 76: 1919.

11. Keith TA, Bader RFW. Chem. Phys. Letters 1993; 210: 223.

12. Steiner E, Fowler PW. J. Phys. Chem. A 2001; 105: 9553.

13. Steiner E, Fowler PW. Chem. Commun. 2001; 2220.

14. Coriani C, Lazzeretti P, Malagoli M, Zanasi R. Theor. Chim. Acta 1994; 89: 181.

15. Zanasi R. J. Chem. Phys. 1996; 105: 1460.

16. Zanasi R, Lazzeretti P, Malagoli M, Piccinini. J. Chem. Phys. 1995; 102: 7150 .

17. Brown RFC, Choi N, Coulston KJ, Eastwood FW, Wiersum UE, Jenneskens LW. Tetrahedron Lett. 1994; 35: 4405.

18. Anderson MA, Brown RFC, Coulston KJ, Eastwood FW, Ward A. Aust. J. Chem. 1990; 43: 1137.
19. Cyrañski MK, Krygowski TM, Katritzky AR, Schleyer PvR. J. Org. Chem. 2002; 67: 1333.

20. Jenneskens LW, Sarobe M, Zwikker JW. Pure Appl. Chem. 1996; 68: 219; Scott LT. Pure Appl. Chem. 1996; 68: 291; Brown RFC, Eastwood FW, Wong NR. Tetrahedron Lett. 1993; 34: 1223.

21. Havenith RWA, Jenneskens LW, Fowler PW, Steiner E. Phys. Chem. Chem. Phys. 2004; 6: 2033.

22. Steiner E, Fowler PW, Havenith RWA. J. Phys. Chem. A 2002; 106: 7048.

23. Steiner E, Fowler PW. Phys. Chem. Chem. Phys. 2004; 6: 261.

24. Gomes JANF. J. Chem. Phys. 1983; 78: 3133.

25. Frost AA, Musulin B. J. Chem. Phys. 1953; 21: 572.

26. Soncini A, Havenith RWA, Fowler PW, Jenneskens LW, Steiner E. J. Org. Chem. 2002; 67: 4753.

27. Havenith RWA, Rassat A, Fowler PW. J. Chem. Soc., Perkin Trans. 2 2002; 723.

28. Fowler PW, Steiner E. J. Phys. Chem. 1997; 101: 1409.

29. Havenith RWA, Lugli F, Fowler PW, Steiner E. J. Phys. Chem. A 2002; 106: 5703

30. Fowler PW, Soncini A. Polycyclic Aromatic Compounds. In press.

31. Steiner E, Fowler PW, Jenneskens LW. Angew. Chem. Int. Ed. 2001; 40: 362.

32. Acocella A, Havenith RWA, Steiner E, Fowler PW, Jenneskens LW. Chem. Phys. Lett. 2002; 363: 64.

33. Steiner E, Fowler PW, Jenneskens LW, Havenith RWA. Eur. J. Org. Chem. 2002; 163.

34. Fowler PW, Steiner E, Acocella A, Jenneskens LW, Havenith RWA. J. Chem. Soc., Perkin Trans. 2 2001; 1058.

35. Guest MF, van Lenthe JH, Kendrick J, Schöffel K, Sherwood P, Harrison RJ. GAMESS-UK, a Package of Ab Initio Programs, 2000; with contributions from Amos RD, Buenker RJ, van Dam HJJ, Dupuis M, Handy NC, Hillier IH, Knowles PJ Bonacic-Koutecky V, von Niessen W, Harrison RJ, Rendell AP, Saunders VR, Stone AJ, Tozer DI, de Vries AH. Derived from the original GAMESS code; Dupuis M, Spangler D, Wendolowski J. NRCC Software Catalog, vol. 1, Program No. QG01 (GAMESS), 1980. CCLRC, Daresbury Laboratory, UK.

36. Lazzeretti P, Zanasi R. SYSMO Package. University of Modena: Modena, 1980; Additional routines, Steiner E, Fowler PW, Havenith RWA.

37. Binsch G, Heilbronner E, Murrell JN. Mol. Phys. 1966; 11: 305. 